\title{
A Review of Morphological and Chemical Properties of Porous Asphalt
}

\author{
N. E Jasni, K. A Masri" and R. P Jaya \\ Department of Civil Engineering, College of Engineering, Universiti Malaysia Pahang, 26300 Gambang, Kuantan, Pahang, Malaysia
}

\begin{abstract}
Porous asphalt mixture is also known as gap graded mixture with less amount of fine aggregate has led the mixture contains high air voids, tends to make the mixture less durable and high porousity. Hence, past researchers has investigate on how to increase the strength of porous asphalt mixture by the addition of additive such as fiber and nanomaterials. The chemical and physical properties of porous asphalt mixture was highlighted in this paper to compare its structure, the bonding between the materials and its chemical composition that exist. This paper reviews on how additive affect the asphalt mixture in terms of Scanning Electron Microscopy (SEM), X-Ray Diffractions (XRD) and Fourier Transform Infrared Spectroscopy (FTIR). These tests are selected to improve the asphalt mixture according to the morphological and chemical properties of porous asphalt. This study is expected to identify the morphological and chemical composition of the materials in asphalt mixture.
\end{abstract}

\section{ARTICLE HISTORY}

Received: $31^{\text {st }}$ July 2021

Revised: $13^{\text {th }}$ Aug 2021

Accepted: 23rd Aug 2021

KEYWORDS
SEM
XRD
FTIR
Asphalt mixture
Fiber

Fiber

\section{INTRODUCTION}

Porous asphalt which is known as the mixture with less fine aggregates has been utilised in late 1970s and is also classified as flexible pavement. Apart from Malaysia, porous asphalt was first used in Australia in 1973 and in 1987 in Japan. A combination of porous asphalt construction provides outstanding service performance for porous asphalt. Air voids aspect is one of the important features of the mixture and it directly influences road performance and pavement service life.

Porous asphalt or known as open graded asphalt is delineated as a hydrocarbon that mix with designated grading and high quality aggregates to supply an asphalt mix with $20 \%$ air voids [1]. Porous asphalt that has been used as the surface layer and dense graded can be differentiate by the surface of the water that can drain out due to its characteristics of high air void structures but it can reduce the function if the pores get clogged. The thickness of the porous asphalt is in between $45 \mathrm{~mm}$ to $55 \mathrm{~mm}$ thick in order to protect the underlying layers against the water penetration over a waterproof layer. Dense layer is usually required under the porous asphalt layer and it is the alternative way to the developers nowadays in solving the storm water management [2].

Porous asphalt, also called porous concrete, allows the rainwater to enter the soil below due to its high porosity. Since the water drained through the pore structure, the stagnant water on road surfaces can be avoided and water conditions have been improved. Porous Asphalt is designed to control the ponding water and also improved the protection during rainfall with low permeability and the water may penetrate into the porous material, thus reducing aquaplaning, ground spray and light in the night to a degree [3].

The adequate affinity between the binder and aggregate also improves resistance to disintegration [4]. Changing the paving structure with the pore structure such as porous asphalt can reduce the noise that came from the friction between traffic loads and the surface. A good qualities of mixtures increase the road surface life span as it received less damage in the course of its life [5].

However, there is some drawbacks of using porous asphalt mixture. A strong gap graded combination configuration bases the pore structure which tends to make the porous asphalt to have low stability and durability due to the high air voids. The problems of high air voids are induced by a deteriorating substances quality, such as ravelling effect as mentioned by Zhang et al. [6]. A type of fault found within the region of contact from pier to pier is a dominant defect in porous asphalt paved which leads to frequent traffic distress such as the rutting [6]. Due to high content of air voids in porous asphalt mixture, binder draindown is regarded as one of the main problems.

Porous constructions are vulnerable to water-induced difficulties in tropical nations such as Malaysia, which gets significant rainfall intensity on a regular basis. Furthermore, rapid development in porous infrastructure and road structure has a significant impact on the durability of porous asphalt layer which are related to some issues of high traffic load. Consequently, the deterioration of asphalt pavement has been accelerated. As a result of these two variables, adhesive and cohesive failure of porous asphalt has leads to stripping as these variables creates bonding loss in binder-aggregate system which deteriorate the pavement performance and service life. The most remarkable problem related to the porous asphalt is its short service time compared with the dense graded asphalt concrete [7]. Also reported by Aman [5], with an air void higher than $20 \%$ in New Zealand in 1980s, porous asphalt is extensively utilised, although it has a short service life. 
Yuniarti [8] mentioned that low pavement strength is another negative side of porous asphalt. The fine aggregate gradation has long been known to affect the mixture's stability. In contrast, less pavement strength and durability with higher coarse aggregate composition that implies more permeability. Compared to dense asphalt mixes, the fine aggregate content and mortar content must be greatly diminished in order to create a high proportion of air voids.

It is a need to investigate the aging impact analysis of porous asphalt mixture on mechanical behaviour and its chemical composition by microstructural analysis. Changes in chemical groups can cause rheological characteristics of asphalt mixtures to vary, risking the mixture to failure. The behaviour of the bitumen as well as the mixture was affected by the microstructure of the fiber content, the pore content of their surfaces and the rigidities. Also, fiber creation must be prevented at all costs and uniform dispersion must be ensured. Herein, analysis and knowledge of structural and compositional features of asphalt mixtures is expected to improve the performance and durability of asphalt pavement at microscopic level.

This study was an attempt to evaluate porous asphalt mixture by examining the air voids content and the properties of asphalt mixture that correlates with permeability of asphalt mixture by using microstructure analysis test. The outcome of this study was to identify the morphological and chemical composition of the materials in asphalt mixture.

\section{RESEARCH FINDINGS}

The microstructure analysis of the fiber has been assessed broadly because it makes a difference to set up the structure, pore substance, thickening branches and evaluating particular surface zones. Allen et al. [9] study revealed that the asphalt microstructure was subjected to some asphalt chemical parameters with strong and observable effect. Hence the microstructure test is needed in order to determine the findings that directly contribute to the improvement of modelling capability, creating new opportunities for enhancement in asphalt binder efficiency and longevity. The effect of aggregate and air voids content in asphalt mixture on the mechanical degradation of asphalt varieties can be studied through microstructure test. The relationship between the micro-mechanics, chemical composition, and macro-mechanical behaviour of bitumen may be investigated by microstructural analysis. The physical activity at high levels is strongly influenced by material contaminator and the morphology of microstructures on small scales. For instance, defects such as cracks and dislocations occur in smaller scales and have a strong influence on macroscale material properties may be detected by microstructural test instead of physical testing.

\section{Scanning Electron Microscopy (SEM)}

In some previous study, morphology, composition, and orientation are included in this electron-sample interaction to extrapolate information about exterior of the particles in details. From Wang et al. [10] SEM was conducted with an additional of Ceramic Fiber (CF). As concluded by researcher, $0.4 \%$ of asphalt mixture were added in the mixture will resulted towards improvement. As shown in Figure 1(a), a tight interlocking bonding between roots of CFs and asphalt which indicate to intense bonding strength between CFs and asphalt. In 2016, Wan et al. [11] researched and concluded that stability and stiffness enhancement will be resulted with mixture of CF fibers compared to asphalt mixture without CFs which lead to different sizes of crack up to $1.9 \mu \mathrm{m}$. Thus, results shows an attribution of CFs fiber ability in connecting cracks and key player in bridging and pulling out activity. In addition, CF characteristic has been pointed out by author through Figure 1(b) which shows CF has the ability in distributing and transfering load from varies directions. Pressure exerted from the aggregates will cause CF to bend and friction will increase between CF and other materials which lead to prevention of relative slippage of aggregates [10].
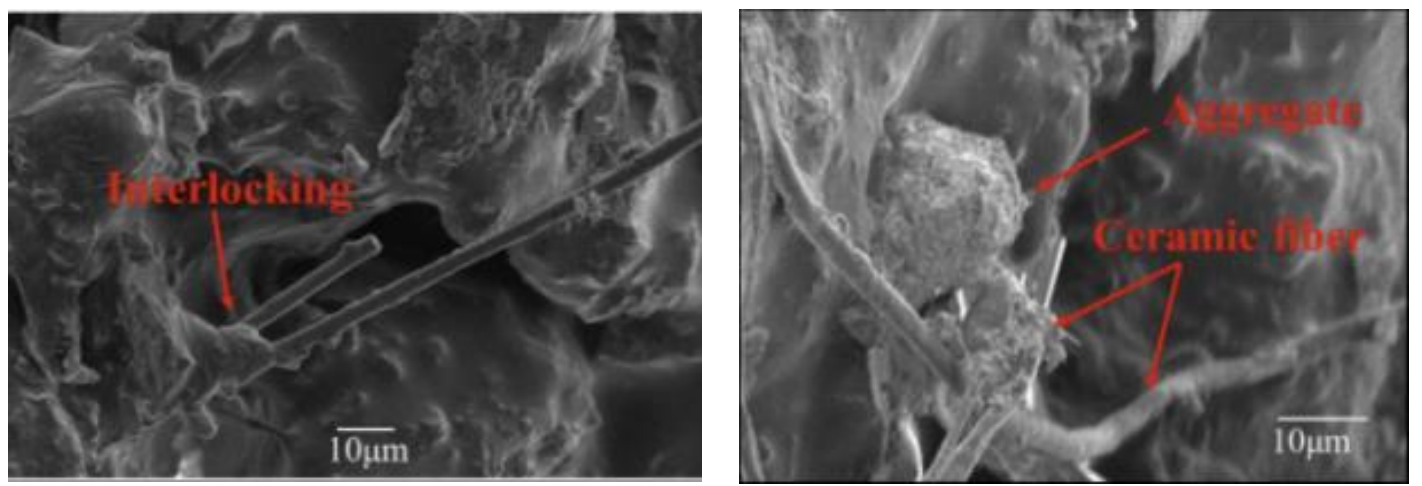

Figure 1. Ceramic Fibers interlocking at roots (a-left) and Ceramic Fibers in asphalt mix (b-right) [10]

On the hand, study reported by Wang et al. [12] this paper studies on chopped basalt fibers in asphalt mixture as the author concluded that $0.3 \%$ of chopped basalt added in the mixture of porous asphalt mixture will resulted to an enhancement of the asphalt mixture integrity and crack propagation hindering. Furthermore, well combination between chopped basalt fiber and porous asphalt mixture contributed to 3-dimensional network structure as the state promptly reinforce the asphalt mixture which explained that fully coated chopped basalt fiber resulted great bonding connection 
when it is mixing with porous asphalt [13]. Past researcher studies shows that the glass wool fibers interaction with aggregates in AC mixtures resulted in good manner as the tensile strength increased and higher durability of the asphalt mixture. As stated, condition proved with good condition of the fibers after an interaction with both aggregates and binder after Indirect Tensile Strength application [14].

\section{X-Ray Diffraction (XRD)}

Past researchers found that natural fiber formed by hybridizing crystalline and amorphous phases. Studies by Luo et al. [15] were observed in two types of fibers, lignin and bagasse fibers. The researchers discovered that crystalline components in celluloses have a peak at $2 \theta=22^{\circ}$, whereas amorphous ingredients such as lignin, hemicelluloses and amorphous cellulose have a peak around $2 \theta=16^{\circ}$ as illustrated in Figure 2 [16]. According to the estimated crystallinity indices, lignin fibers have a greater order of cellulose crystals along the fibers axis than bagasse fibers with $67.2 \%$ and for lignin fibers at $72.2 \%$ as in Figure 2. This is due to the production of lignin fibers, the greater lignin fiber outcome is attributale to the bleaching and chemical treatment [17].

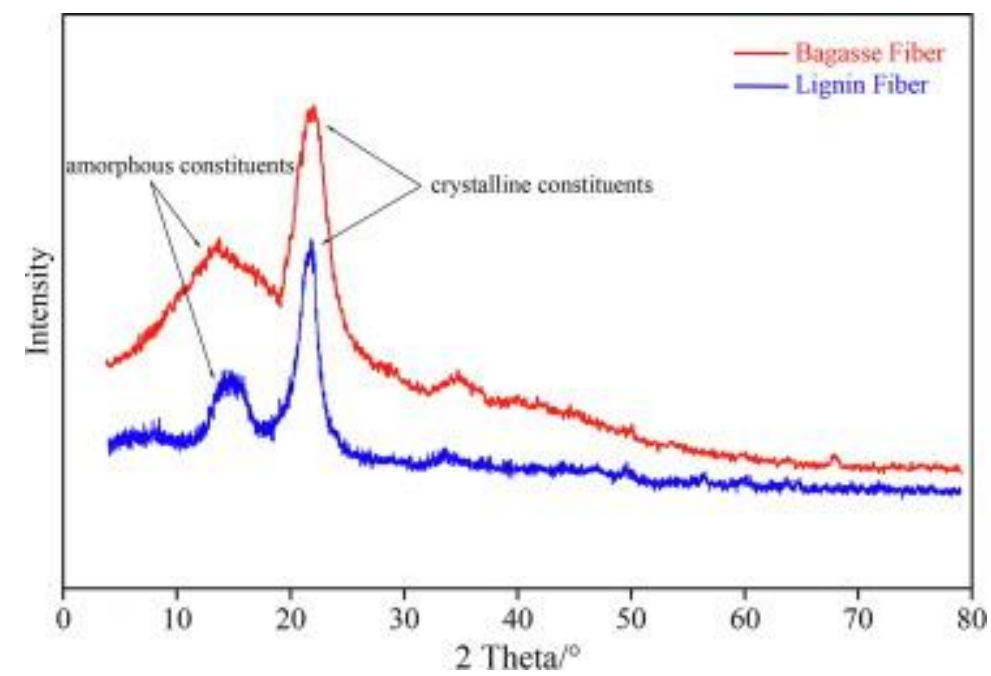

Figure 2. XRD peaks of bagasse fiber and lignin fiber [17]

Study by Mubaraki [18] found that, the use of modifiers in varied percentages of modifier absorption, Acrylate Styrene Acrylonhrtilrei (ASA) polymer and nanomaterials of $\mathrm{Al}_{2} \mathrm{O}_{3}$ binders are still amorphous to the original asphalt, gives the conclusion to raising the absorption of ASA polymer may increasing the proportion of crystalline phase. On the other hand, the use of coconut shell fiber in the study by Ting et al. [19] it was found that element of Dy, Xe, Pr, and Eu of the coconut fiber does not exist, which this make the asphalt mixture is free from radioactive materials.

\section{Fourier Transform Infrared Spectroscopy (FTIR)}

According to Hofko et al. [20] the spectrum analysis derived from FTIR, multiple classes of molecular limits can be easily identified by the wavelength of the absorption spectrum clearly defined by the characteristic band maxima. Studies by $\mathrm{Yu}$ et al. [21] as in Figure 3, at $813 \mathrm{~cm}-1 \mathrm{~m}$ modified bamboo fibers (BF) containing melamine-formaldehyde exhibit a unique feature. This is due to the $\mathrm{N}$-containing heterocyclic ring out of the bending vibration $-\mathrm{N}=\mathrm{C}-\mathrm{H}$ [22]. Other than that, past study by Liu et al. [23] pointed out that the distinctive signal at $1335 \mathrm{~cm}-1$ corresponding to the stretching vibration of the phenolic -OH group from the unmodified fibers vanished in the spectra of the MF-treated BFs, indicating that the F copolymer interacted with the $-\mathrm{OH}$ groups on the surface of the BFs. 


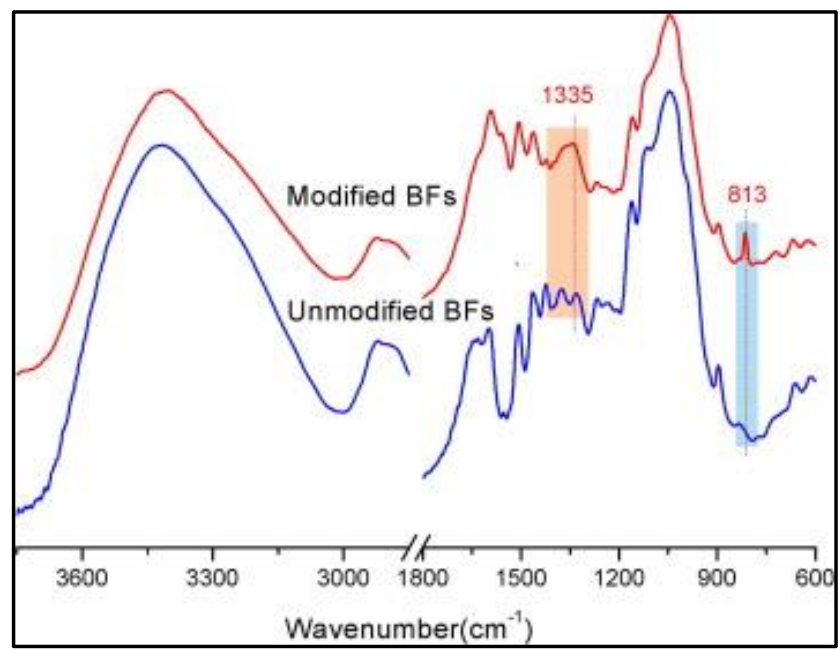

Figure 3. Spectrum analysis of bamboo fibers [21]

Mubaraki [18] studied the amount of asphalt binder using ASA polymer and $\mathrm{Al}_{2} \mathrm{O}_{3}$ nanomaterials in asphalt mixture and found that $4 \%$ can considered as optimum percentage of modifier for both types. Despite their greatest points, the FTIR spectra of ASA polymer and $\mathrm{Al}_{2} \mathrm{O}_{3}$-altered asphalt binders remained identical, indicating that no structural changes occurred in the altered binders connected with the conventional asphalt binder. From Ting et al. [19] this study evaluated coconut shell in asphalt mixture and conclude that the raw materials of coconut shell tend to give better performance towards asphalt mixture in field. The researchers [24] come out with conclusion that mullite, quartz, carbon and vitreous phases are confirmed presence because of the series of bands of mullite, quartz and the glassy phase of the ash intersect in the region among $1200 \mathrm{~cm}-1$ and $1434.12 \mathrm{~cm}-1$.

\section{CONCLUSION}

This paper reviews a modern experimental instruments to investigate the inner structure of asphalt mixture. Investigations included the morphological and chemical properties of asphalt mixture with the additives in order to find out the structure and chemical that exist in mixture. Through the reviews that has been made, almost every additive has it own specialist to make the mixture better especially with the addition of fibers. Fibers tends to make amount of air voids reduce since the air voids has been filled with fibers in the mixture. The additives also seems to have a great bonding with other materials in the mixture and this may constribute to the use in construction field.

\section{ACKNOWLEDGEMENT}

The authors wish to acknowledge the support provided by University Malaysia Pahang for funding this paper under internal research grant number PGRS200374.

\section{REFERENCES}

[1] Ma, X., et al., Performance of porous asphalt mixture with various additives. International Journal of Pavement Engineering, 2018. 19(4): p. 355-361.

[2] Sharma, A., Satyender, and D.K. Sharma, Laboratory Performance of Porous Asphalt Pavement. 2017. p. 1-9.

[3] Afonso, M.L., D.A. Marisa, and C.S. Fael, Study of the porous asphalt performance with cellulosic fibres. Construction and Building Materials, 2017. 135: p. 104-111.

[4] Aman, M.Y., Z. Shahadan, and N.R.M. Tamin, A comparative study on properties of Malaysian porous asphalt mixes with different bitumen contents. Research Journal of Applied Sciences, Engineering and Technology, 2015. 9(10): p. 797-806.

[5] Aman, M.Y., Water sensitivity of warm porous asphalt incorporating Sasobit. 2013, Universiti Sains Malaysia.

[6] Zhang, H., et al., Performance enhancement of porous asphalt pavement using red mud as alternative filler. Construction and Building Materials, 2018. 160: p. 707-713.

[7] Qian, Z. and Q. Lu, Design and laboratory evaluation of small particle porous epoxy asphalt surface mixture for roadway pavements. Construction and Building Materials, 2015. 77: p. 110-116.

[8] Yuniarti, R., Resistance to degradation of porous asphalt mixture using pine resin as asphalt modifier. Jordan Journal of Civil Engineering, 2019. 13(1).

[9] Allen, R.G., et al., The effects of chemical composition on asphalt microstructure and their association to pavement performance. International Journal of Pavement Engineering, 2014. 15(1): p. 9-22. 
[10] Wang, X., et al., Investigation of the Performance of Ceramic Fiber Modified Asphalt Mixture. Advances in Civil Engineering, 2021. 2021.

[11] Wan, J., et al., Characteristics of ceramic fiber modified asphalt mortar. Materials, 2016. 9(9): p. 788.

[12] Wang, S., et al., Investigating the effects of chopped basalt fiber on the performance of porous asphalt mixture. Advances in Materials Science and Engineering, 2019. 2019.

[13] Tanzadeh, R., J. Tanzadeh, and S.A. Tahami, Experimental study on the effect of basalt and glass fibers on behavior of opengraded friction course asphalt modified with nano-silica. Construction and Building Materials, 2019. 212: p. 467-475.

[14] Mrema, A.H., et al., Performance of Glass Wool Fibers in Asphalt Concrete Mixtures. Materials, 2020. 13(21): p. 4699.

[15] Luo, D., et al., The performance of asphalt mixtures modified with lignin fiber and glass fiber: A review. Construction and Building Materials, 2019. 209: p. 377-387.

[16] Jebadurai, S.G., et al., Comprehensive characterization of natural cellulosic fiber from Coccinia grandis stem. Carbohydrate polymers, 2019. 207: p. 675-683.

[17] Li, Z., et al., Investigation on characteristics and properties of bagasse fibers: Performances of asphalt mixtures with bagasse fibers. Construction and Building Materials, 2020. 248: p. 118648.

[18] Mubaraki, M., The Effect of Modified Asphalt Binders by Fourier Transform Infrared Spectroscopy, X-Ray Diffraction, and Scanning Electron Microscopy. Journal of Materials and Engineering Structures «JMES», 2019. 6(1): p. 5-14.

[19] Ting, T.L., et al., A review of chemical and physical properties of coconut shell in asphalt mixture. Jurnal Teknologi, 2016. 78(4).

[20] Hofko, B., et al., Repeatability and sensitivity of FTIR ATR spectral analysis methods for bituminous binders. Materials and Structures, 2017. 50(3): p. 187.

[21] Yu, D., et al., Preparation and mechanical properties of asphalt mixtures reinforced by modified bamboo fibers. Construction and Building Materials, 2021. 286: p. 122984.

[22] Cesar, T., et al., Melamine polymerization in organic solutions and waterlogged archaeological wood studied by FTIR spectroscopy. Journal of Cultural Heritage, 2017. 23: p. 106-110.

[23] Liu, W., T. Xie, and R. Qiu, Bamboo fibers grafted with a soybean-oil-based monomer for its unsaturated polyester composites. Cellulose, 2016. 23(4): p. 2501-2513.

[24] Madakson, P., D. Yawas, and A. Apasi, Characterization of coconut shell ash for potential utilization in metal matrix composites for automotive applications. International journal of engineering science and technology, 2012. 4(3): p. 1190-1198. 